Differences in limb coordination in polyrhythmic production among water polo players, artistic swimmers and drummers

Ravisara Vathagavorakul ${ }^{1}$, Tomohiro Gonjo ${ }^{2}$, Miwako Homma ${ }^{1}$

${ }^{1}$ Faculty of Health and Sport Sciences, University of Tsukuba, Japan

${ }^{2}$ Department of Physical Performance, Norwegian School of Sport Sciences, Norway

Corresponding author:

Ravisara Vathagavorakul

Faculty of Health and Sport Sciences

University of Tsukuba

Tennodai 1-1-1, Tsukuba, Japan

Phone: +81298532670

e-mail:s1630476@s.tsukuba.ac.jp

190 words (Abstract)

3620 words (Main text: Introduction-Conclusion) 


\section{Differences in limb coordination in polyrhythmic production among water polo players, artistic swimmers and drummers}

This study compares polyrhythmic production ability between water polo players (WPs), artistic swimmers (ASs) and drummers (Ds), to assess how their differing experiences in coordinating complex inter-limb activity with music affected this ability. Eight ASs, eight WPs and eight Ds participated. They were asked to perform finger and foot taps in a single-rhythm task (every $750 \mathrm{~ms}$ ) and two polyrhythmic tasks (finger and foot taps at 750 and $500 \mathrm{~ms}$, respectively, and vice versa). The percentage of correct response cycles (PCRC), subjective difficulty scores were collected and analysed using a two-way mixed analysis of variance (ANOVA) and coefficients of variation of the inter-tap interval $\left(\mathrm{CV}_{\text {ITI }}\right)$ were collected and analysed using a three-way mixed analysis of variance (ANOVA). The three groups showed no statistically significant differences in the single-rhythm task. However, on polyrhythmic tasks, the WPs were significantly outperformed

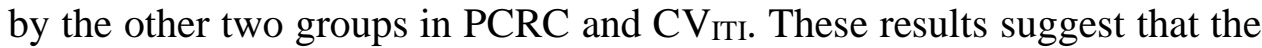
experience of coordinating limbs with music has positive impacts on polyrhythmic production ability. They also imply that ASs and Ds have similar polyrhythmic production ability despite the apparent differences in task complexity in their daily training and performances.

Keywords: polyrhythm; limb coordination; artistic swimming; tapping 


\section{Introduction}

Polyrhythmic tasks have been a popular research topic, since they are difficult to perform and require completing multiple tasks simultaneously with different rhythms (Klapp, 1979; Kurtz \& Lee, 2003; Peper \& Beek, 1998; Starke \& Baber, 2017; Summers \& Kennedy, 1992; Summers, Rosenbaum, Burns, \& Ford, 1993; Summers, Todd, \& Kim, 1993). Such tasks entail the concurrent production of many conflicting but isochronous motor sequences (Summers, Ford, \& Todd, 1993; Tajima \& Choshi, 2000). For example, the 3:2 (three against two) polyrhythmic pattern involves the coordination of three taps with one limb and two taps with another limb during each cycle. Successful polyrhythmic performance requires good musical cognition as well as movement control (Yokus \& Yokus, 2015).

Polyrhythmic skill combines limb coordination and sensorimotor synchronisation abilities so as to coordinate movements with an external rhythm (Repp \& Su, 2013). Good limb coordination is essential to achieve the desired movement outcome. Studies have shown that professional or experienced musicians, dancers and athletes outperform amateur or inexperienced groups in limb coordination (Martins, Neves, Rodrigues, Vasconcelos, \& Castro, 2018; Mo \& Chow, 2018; Seifert, Leblanc, Chollet, \& Delignières, 2010; Seifert et al., 2011). Similarly, research has found that experienced musicians and dancers have better sensorimotor synchronisation ability than their less experienced counterparts (Jin et al., 2019; Karpati, Giacosa, Foster, Penhune, \& Hyde, 2016; Repp, 2010; Sommer, Hager, Boraxbekk, \& Ronnqvist, 2018). However, few studies have addressed sensorimotor synchronisation in athletes (Buhmann, Moens, Van 
Dyck, Dotov, \& Leman, 2018; Maes, Lorenzoni, \& Six, 2018), even though athletes in various sports may benefit from sensorimotor synchronisation (van de Rijt, 2018).

Although polyrhythms are counter-intuitive and performing them in consistent timing is challenging (Clayton, Sager, \& Will, 2004; Peper \& Beek, 1998), studies have shown that highly skilled musicians can perform polyrhythmic tasks better than nonmusicians. Among musicians, drummers, who are usually trained to coordinate all four limbs in different external rhythms, tend to have better limb coordination than other types of musicians (Krause, Pollok, \& Schnitzler, 2010). These findings indicate that the ability to perform polyrhythmic tasks can be improved with extensive training (Deutsch, 1978, 1996; Kurtz \& Lee, 2003; Peper \& Beek, 1998; Shaffer, 1981; Summers, Rosenbaum, et al., 1993). Park and Sternad (2015) studied how young adults acquired and retained bimanual polyrhythmic ability. Their results showed participants with musical background outperformed the other participants. However, due to a small number of participants with musical backgrounds (2 from 16), the study was inconclusive.

Polyrhythmic skill is essential in some sports (Jagacinski, Kim, \& Lavender, 2009). Artistic swimming and water polo require athletes to perform two very different categories of motor sequences: some above and others under the water. The objective of the underwater movements is to provide a solid foundation for the above-water actions that are essential for success. In artistic swimming, above-water movements constitute the main performance, combining artistry and technical difficulty in synchronisation with music, and are scored by judges. Similarly, the above-water movements in water polo pertain to shooting, passing, blocking and defending. Thus, in both sports the lower and upper extremities must move independently. For this reason, these athletes are accustomed to completing exercises that involve complex inter-limb coordination. 
Although drummers (Ds), artistic swimmers (ASs) and water polo players (WPs) have extensive experience in limb coordination, they have very different experiences in coordinating their movements with external rhythms such as music. Among the three groups, Ds have the most extensive experience in coordinating their movements with music, since they must move all four limbs independently yet in coordination with a musical rhythm, making this activity very demanding physically (Bianco, Berchicci, Perri, Quinzi, \& Di Russo, 2017). ASs move the upper and lower extremities independently, but only one pair of limbs at a time (either the upper or lower extremities) must be coordinated with the music. Similar to ASs, WPs move their upper and lower limbs independently, but they are not required to coordinate any limb movements with music.

Although polyrhythmic performance has been widely studied among musicians and specifically Ds, we know much less about this capacity among athletes. Several studies have assessed polyrhythm using bimanual tasks. However, polyrhythmic production which requires a coordinated movement of different limbs with different rhythms has not been studied even though it is an essential skill in several sports such as aesthetic swimmers. Since rhythmic tapping requires accurate and precise movements, both sides of corpus callosum, which are required to successfully produce polyrhythmic bimanual tasks, need to be activated to produce fine and delicate movements to match tapping with rhythms (Beaulé, Tremblay, \& Théoret, 2012; Vuust, Wallentin, Mouridsen, Ostergaard, \& Roepstorff, 2011). Hence, the neurophysiological mechanism of bimanual tasks should be similar to rhythmic tapping tasks. Comparing polyrhythmic limb coordination between groups of musicians and athletes would provide more information on how coordinating limb movements with music affects polyrhythmic performance. 
Therefore, the purpose of this study was to compare polyrhythmic limb coordination between Ds, ASs and WPs.

\section{Methods}

\section{Participants}

Three groups of highly well-trained participants volunteered to participate in this study. All participants had at least six times a week for two years of regular practice. The ASs (eight females; mean age $23.1 \pm 2.9$ years) had participated in international and national competitions and averaged $13.25 \pm 2.5$ years of artistic swimming experience. The WPs (eight males; mean age $20.75 \pm 2.4$ years) had $9.75 \pm 2.1$ years of water polo experience. Both of these groups, on average, had less than two years of musical experience, defined as experience in learning singing or a musical instrument (WPs, $1.3 \pm 1.6$ years; ASs, 1.9 \pm 3.2 years). Finally, the Ds (six males and two females; mean age $21.6 \pm 3.8$ years) had played percussion instruments for an average of $10 \pm 2.8$ years. The testing procedures were approved by the University of Tsukuba Ethics Committee, and each participant signed an informed consent form.

\section{Experimental Tasks}

The participants were required to perform finger- and foot-tapping tasks, tapping in time with a metronome beat. Of the three tasks, one involved tapping with a single rhythm (every $750 \mathrm{~ms}$ with both finger and foot); the other two were polyrhythmic (tap every 500 ms with the finger and every $750 \mathrm{~ms}$ with the foot, or vice versa). All tapping was done 
on percussion pads (SPD ONE Percussion, Roland Corporation, Shizuoka, Japan). To avoid the effect of auditory feedback, participants did not hear any sound except for the sound of the finger or foot hitting the pad. The metronome ticking interval was set at 750 ms in every trial. In the single-rhythm task (750:750), the participants were instructed to perform both finger and foot tapping in synchronisation with the metronome beat. In the 750:500 polyrhythm task, they were required to perform a finger tap every $750 \mathrm{~ms}$ with the metronome, but a foot tap every $500 \mathrm{~ms}$ (or three times for every two ticks of the metronome). In the 500:750 polyrhythm task, the participants attempted to perform a finger tap every $500 \mathrm{~ms}$, but a foot tap every $750 \mathrm{~ms}$. Table 1 summarizes the three task descriptions contained in the experiment.

Table 1. Description of tasks performed by the participants

\begin{tabular}{|l|l|l|l|l|}
\hline & Metronome & Finger & Foot & Types of rhythm \\
\hline Task 1 & $750 \mathrm{~ms}$ & $750 \mathrm{~ms}$ & $750 \mathrm{~ms}$ & Single rhythm \\
\hline Task 2 & $750 \mathrm{~ms}$ & $750 \mathrm{~ms}$ & $500 \mathrm{~ms}$ & \multirow{2}{*}{ Polyrhythm } \\
\hline Task 3 & $750 \mathrm{~ms}$ & $500 \mathrm{~ms}$ & $750 \mathrm{~ms}$ & \\
\hline
\end{tabular}

\section{Design and Procedure}

The participants performed a total of six trials (two on each of the three tasks). Upon arriving, they received instruction on the tasks. The participants were instructed to sit on a chair with wearing a headphone, and the percussion pads were located in front of the participants (Figure1). Each participant was required to perform six trials (three tasks $\mathrm{x}$ two trials per task). The three tasks were single rhythm (750:750) and two polyrhythms 
(750:500 and 500:750). The participants repeated each task twice. The average of two trials was then used to perform further analyses. They were granted 5 minutes to practice each task before the trials began, or 15 minutes of total practice. The order of trials was fully randomised. The participants were asked to begin their first tap on the fifth beat and to continue tapping for $20 \mathrm{~s}(20,000 \mathrm{~ms})$. They were instructed to continue tapping without stopping until the end of the trial. The participants were granted a short break after each trial.

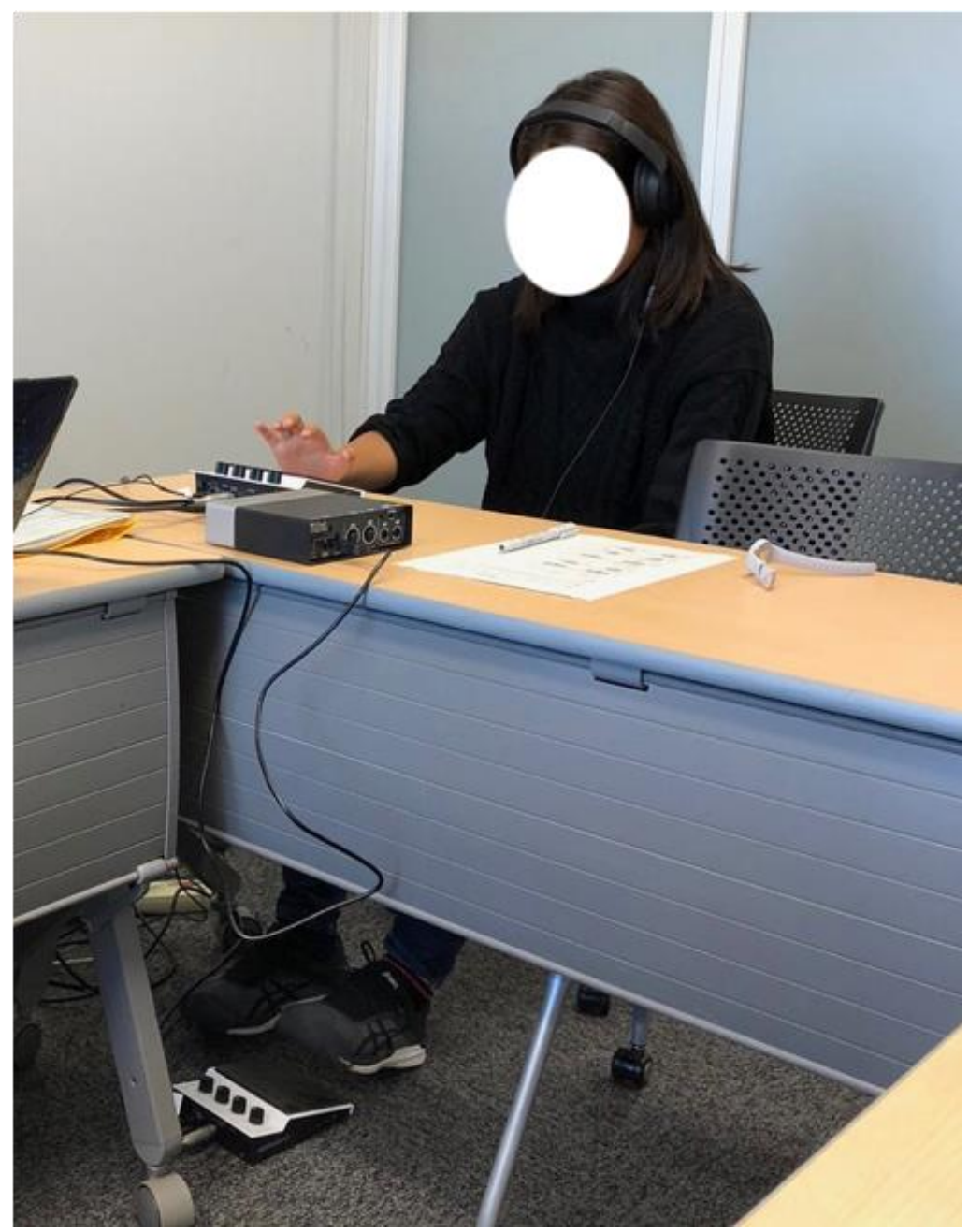

FIGURE 1. Experimental setting. Each participant was seated on a chair and tap his/her finger and foot on percussion pad. 


\section{Data Collection and Analysis}

All tasks were recorded using Logic Pro X (Apple Inc., Cupertino, California, USA) at a sampling frequency of $44,100 \mathrm{~Hz}$. They were converted to a digital audio file (.wav format) with the same sampling frequency. Once all files (tapping responses and metronome beats) had been converted to audio files, MATLAB (R2017b, MathWorks, Inc., Natick, Massachusetts, USA) was used to analyse the signals. Audio data were smoothed using a bidirectional second-order low-pass filter (cut-off frequency $=150 \mathrm{~Hz}$ ). After filtering, the onsets of tapping and the metronome beats were detected. Twentyfour response cycles in the single-rhythm task and 12 response cycles in the polyrhythmic tasks were analysed for each participant trial, and the percentage of correct response cycles (PCRC; the ratio between the number of correct response cycles and the total number of cycles analysed) was calculated, based on Summers and Kennedy (1992); Summers, Rosenbaum, et al. (1993). A response cycle was deemed correct if (1) all taps and metronome beats were in the same pattern (Figure 2), and (2) taps intended to be performed in synchronisation with metronome beats were conducted within $20 \mathrm{~ms}$ of the associated metronome beat.

The inter-tap interval (ITI) was also calculated. ITI provided absolute timing measurement but gave no information on how the participants performed their tapping relative to the given metronome beats. Therefore, the coefficient of variation of ITI $\left(\mathrm{CV}_{\mathrm{ITI}}\right)$ was calculated from the ratio between the mean and standard deviation. Moreover, a subjective difficulty score (SDS) was obtained by asking participants to rate the difficulty of each task, on a scale from 0 (easiest) to 10 (hardest), after each trial. 
750:750 Single rhythm task

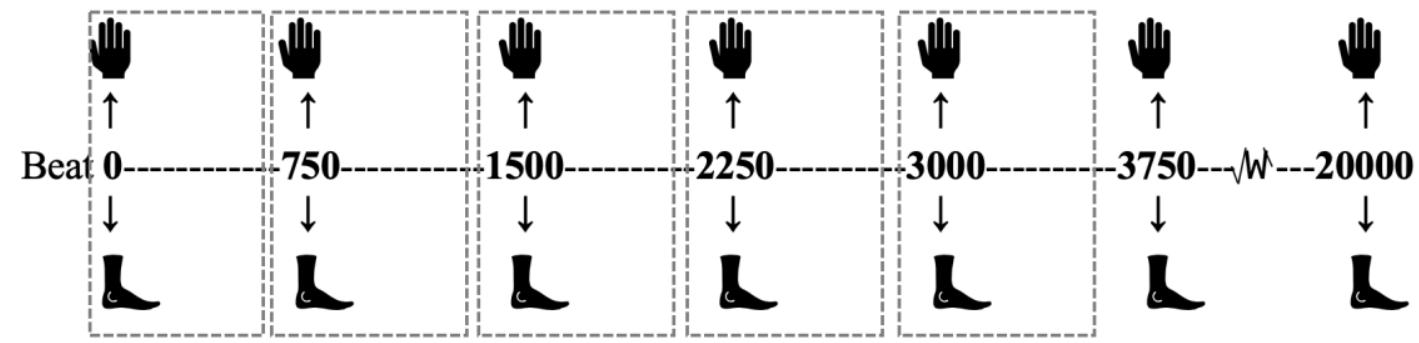

750:500 polyrhythm task

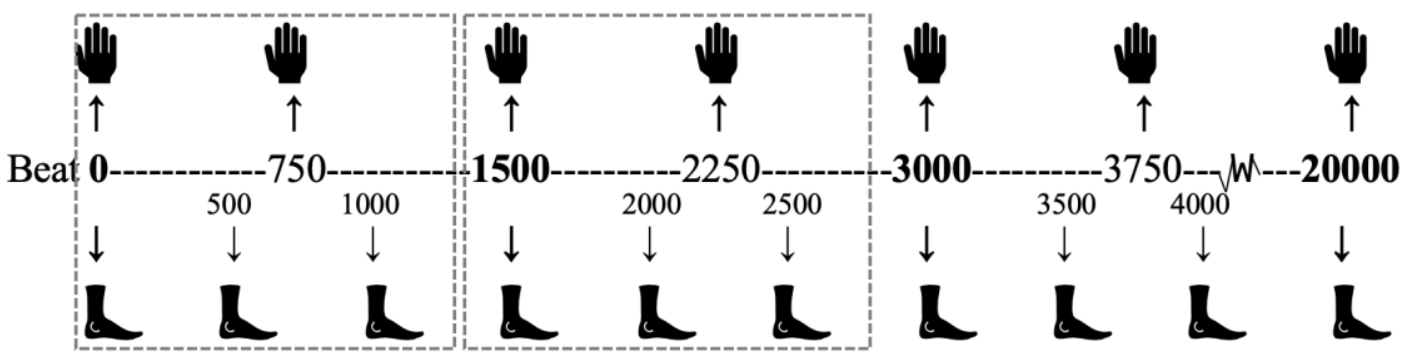

500:700 polyrhythm task

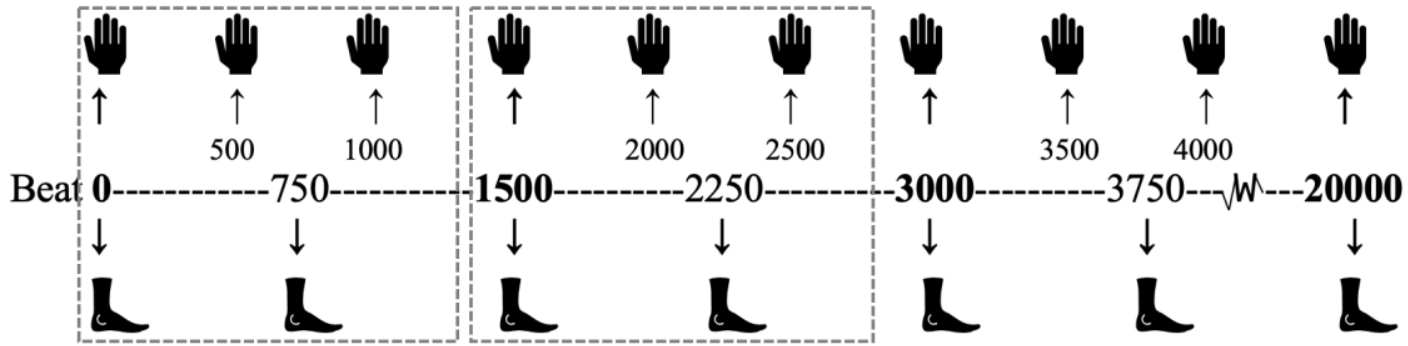

FIGURE 2. Pattern of 750:750 single rhythm, 750:500, and 500:750 polyrhythm tasks with a cycle duration of $20 \mathrm{~s}(20000 \mathrm{~ms})$. Dash-line boxes show examples of one response cycle in each task. 


\section{Statistical Analysis}

A two-way mixed-design analysis of variance (ANOVA) was performed to assess differences in PCRC and SDS between the groups, with the type of rhythm (750:750 single rhythm, 750:500 and 500:750 polyrhythms) as a within-subjects factor and group (AS, WP, or D) as a between-subjects factor. A three-way mixed-design ANOVA was performed on $\mathrm{CV}_{\text {ITI}}$, with the type of limbs (finger versus foot) and type of rhythm (750:750, 750:500, or 500:750) as two within-subjects factors and group (AS, WP and D) as a between-subjects factor. The Greenhouse-Geisser correction was used when Mauchly's test of sphericity was significant. Because some factors (group and rhythm) had more than two levels, a one-way ANOVA was also performed as a post hoc analysis if the statistical difference was found. Partial eta squared was calculated and interpreted in accordance with Cohen, Cohen, West, and Aiken (2003) where small, medium, and large thresholds were $.01, .06$, and .14, respectively. All analyses were performed in IBM SPSS Statistics version 24 (IBM, NY, USA).

\section{Results}

In this section, we present the results obtained by means of the ANOVA tests for each variable. An overview of the ANOVA tests (indicating the main effect of group, rhythm and limb on each variable) is presented in Table 2. 
Table 2. Interactions and main effects of each variable and tasks

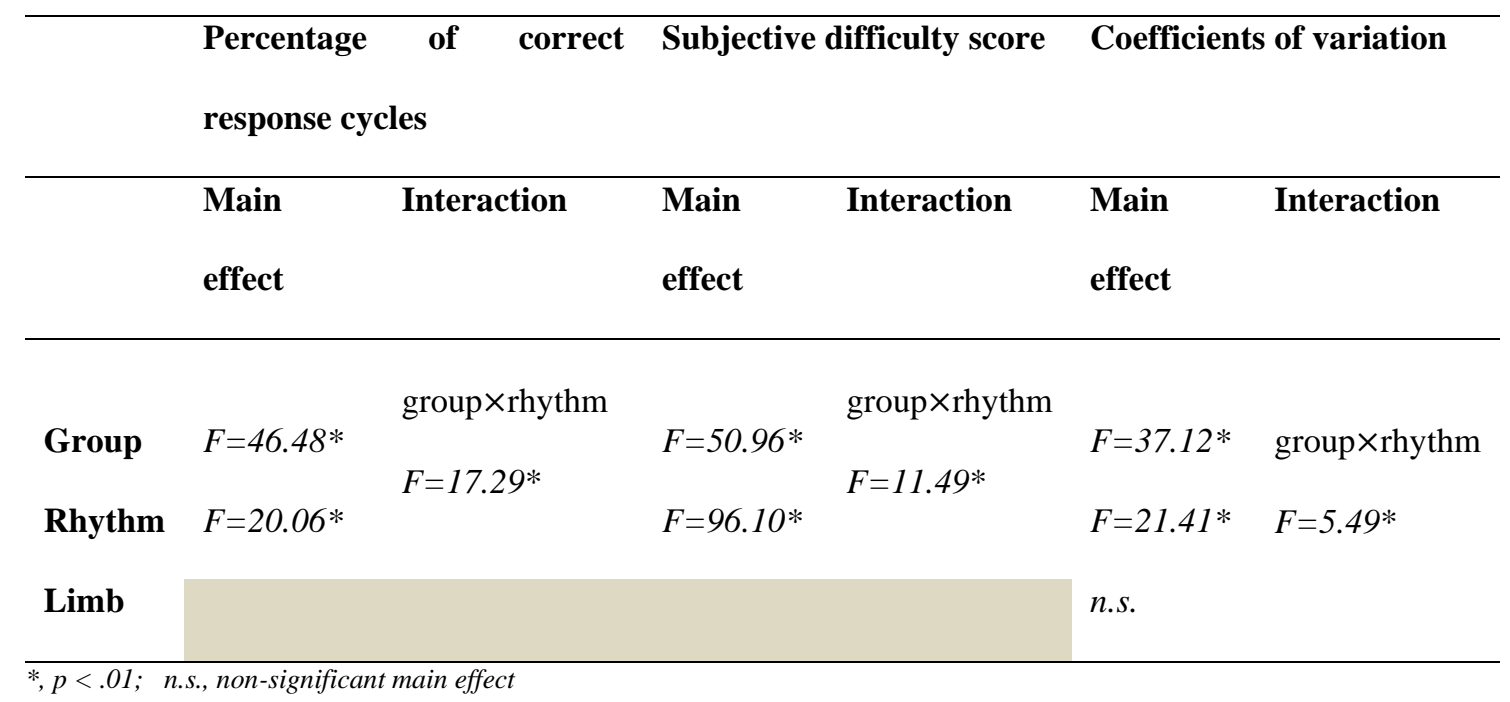

\section{Percentage of Correct Response Cycles}

Figure 3 shows PCRC as a function of the type of rhythm. There was an interaction between group and rhythm tasks $(F=12.176, p<.001)$. The effect sizes of group and rhythm tasks were large ( 0.736 and 0.576 , respectively). The post hoc analysis of the two main effects showed that the PCRC among WPs was statistically lower than that among ASs and Ds, whereas no statistical difference was found between the ASs and Ds ( $p=$ $.21)$. On the other hand, the SD of Ds was $1 \%$ of the SD of ASs.

The participants performed the single-rhythm task significantly better than either polyrhythm task; performance on the two polyrhythmic tasks did not differ significantly $(p=.99)$. The PCRC for the WPs was significantly lower than that for the ASs and Ds in both the 750:500 and 500:750 rhythms ( $p<.001)$. 


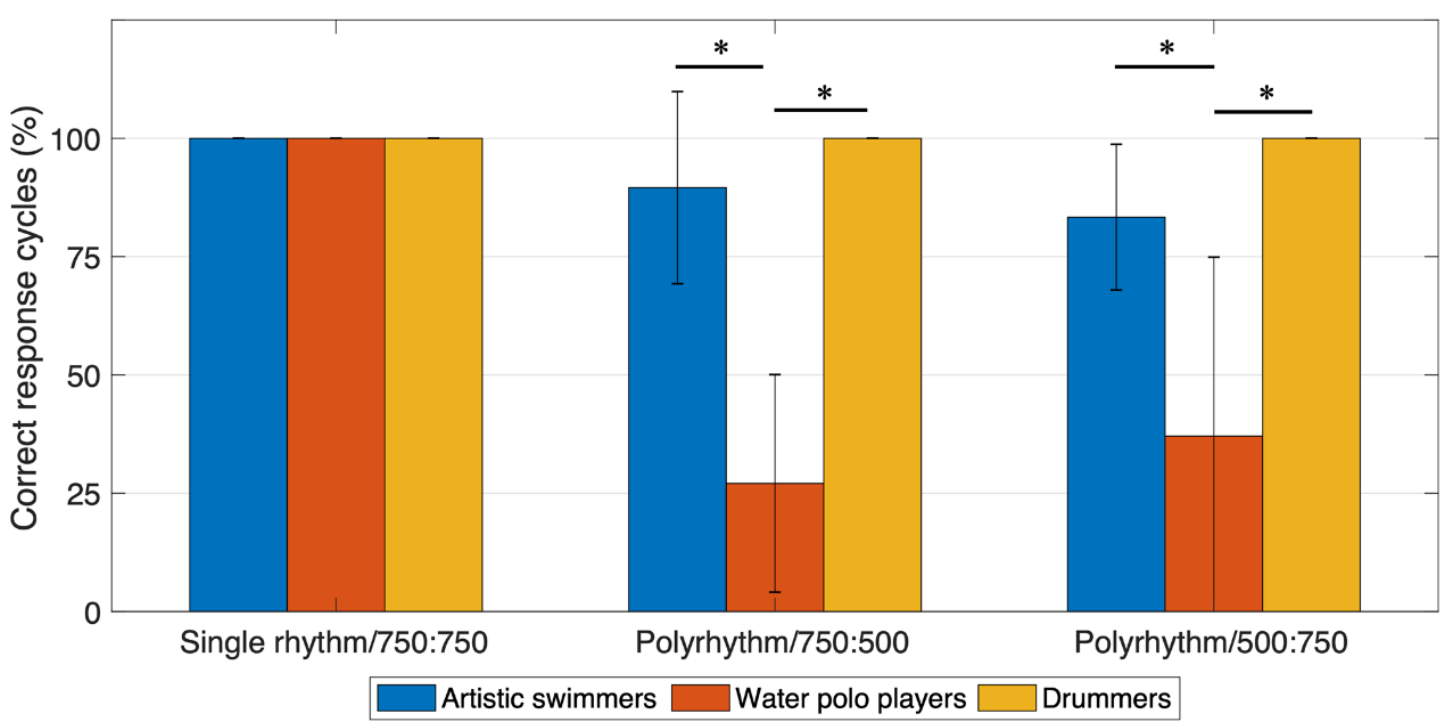

FIGURE 3. Percentages of correct responses cycles for three rhythm tasks. Vertical bars represent between subject standard deviation. *significant difference with group, $\mathrm{p}<.01$

\section{Subjective Difficulty Scores}

Figure 4 displays SDS as a function of rhythm types. A two-way ANOVA analysis showed an interaction between group and rhythm tasks $(F=8.356, p<.001)$. The effect sizes of groups and rhythm tasks were large (0.758 and 0.863 , respectively). Post hoc analysis for groups and polyrhythms showed that the SDS reported by the Ds was significantly lower than that among the ASs or WPs (both $p<.001$ ). All participants described the polyrhythmic tasks (750:500 and 500:750) as more difficult than the singlerhythm task. However, no statistical difference was found in SDS between the 750:500 and 500:750 tasks. A one-way ANOVA showed that the SDS of Ds was significantly lower than that among ASs and Ds in both the 750:500 and 500:750 rhythms $(p<.001)$, but no difference in SDS between groups was found on the single-rhythm task. 


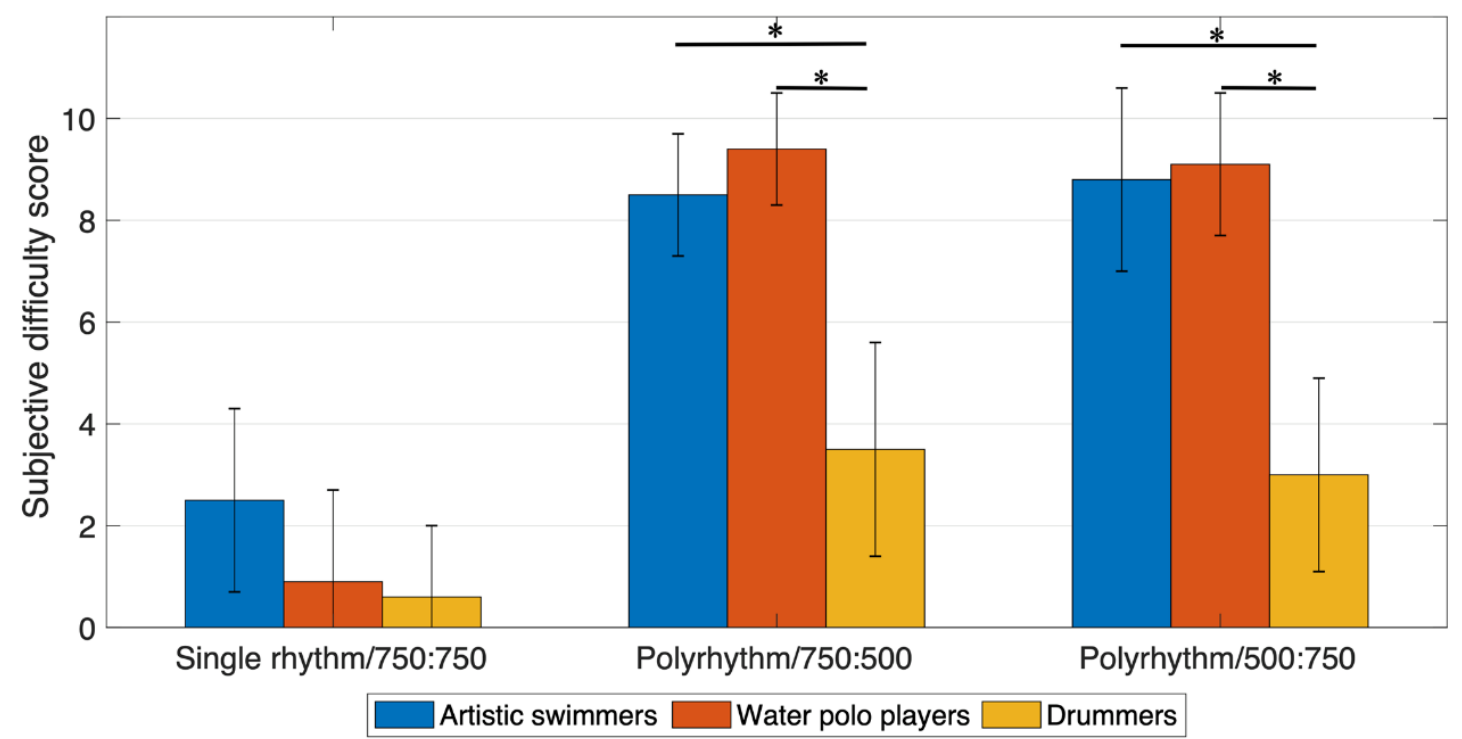

FIGURE 4. Mean subjective difficulty score for three types of rhythms. Vertical bars represent between subject standard deviation. *significant difference with group, $\mathrm{p}<.01$.

\section{Coefficients of Variation of the Inter-tap Interval}

Figure 5 illustrates the mean $\mathrm{CV}_{\text {ITI }}$ as a function of rhythm type, and Table 3 shows the mean ITI for each group, limb, and rhythm tasks. A three-way ANOVA was performed, and the results showed that there was no three-way interaction among the three main factors $(F=.671, p=.614)$; only one two-way interaction, between group and rhythm, was significant $(F=8.271, p=.006)$. The effect sizes of group, limb, and rhythm tasks, were $0.608,0.006$, and 0.404 , respectively, showing that the effect was large in the group and rhythm, but small in the limb. Post hoc analysis showed that all three inter-group comparisons regarding $\mathrm{CV}_{\text {ITI }}$ (ASs versus WPs, ASs versus Ds and WPs versus Ds) yielded significant differences. The SD of Ds was $13 \%$ of the SD of ASs and $6 \%$ of the 
SD of WPs. On the polyrhythmic 750:500 task, WPs had a higher $\mathrm{CV}_{\text {ITI }}$ than ASs and Ds; on the 500:750 task, WPs' performance was significantly lower than that of Ds but not of ASs. As for the rhythm main effect, the results showed that the $\mathrm{CV}_{\text {ITI }}$ on the singlerhythm task was lower than that on the two polyrhythmic tasks, but there was no statistically significant difference in $\mathrm{CV}_{\text {ITI }}$ between the polyrhythmic tasks.

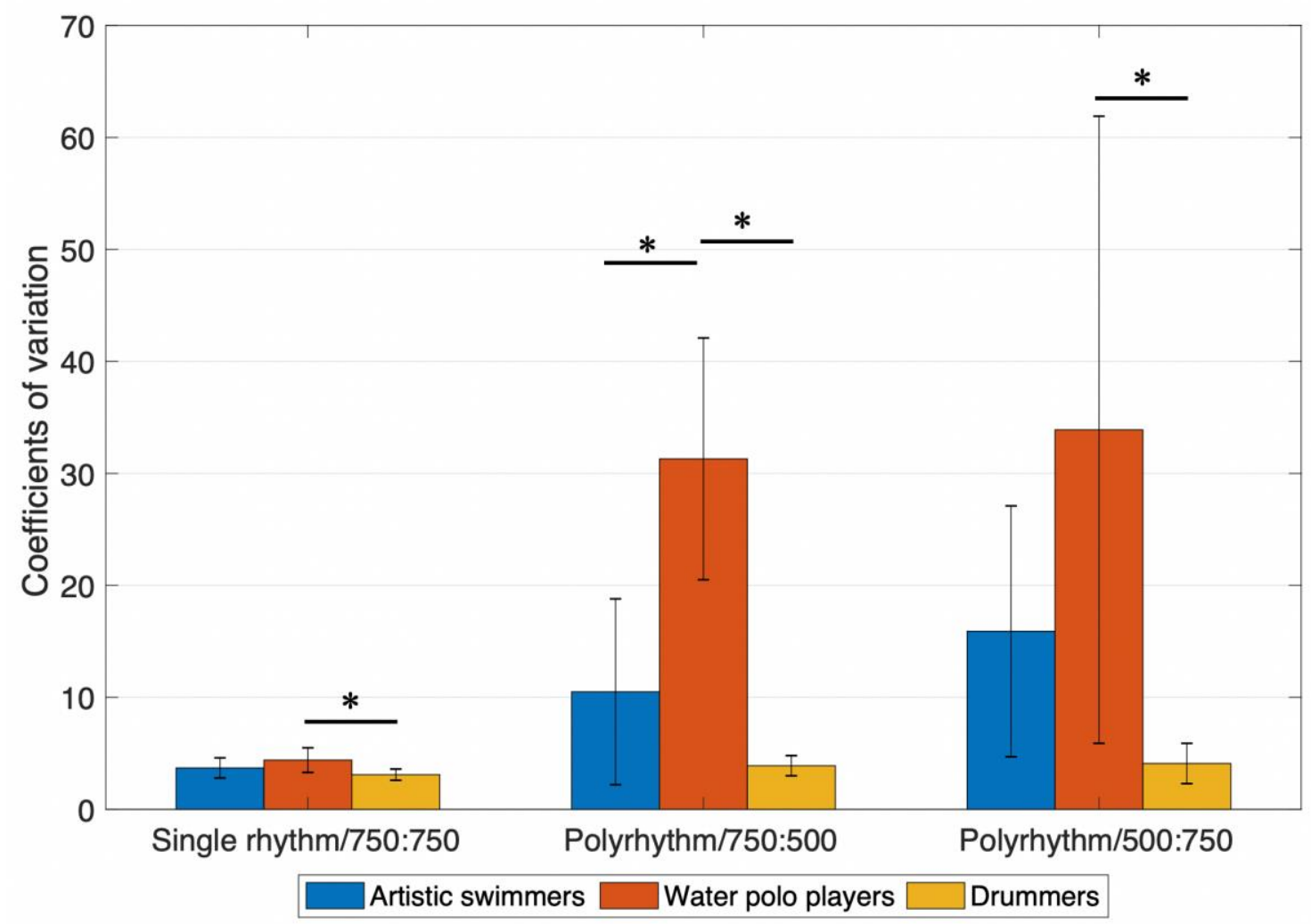

FIGURE 5. Mean coefficients of variation of the inter tapping interval for finger and foot. Vertical bars represent between-subject standard deviation. *|significant difference with group, $\mathrm{p}<.01$. 
Table 3. Mean cycle durations (in milliseconds) in the tapping tasks

\begin{tabular}{cccc}
\hline Tapping tasks & Artistic swimmers & Water polo players & Drummers \\
\hline 750:750 Single rhythm & & & \\
Finger (750ms) & 749.4 & 746.7 & 748.6 \\
Foot (750ms) & 748.4 & 746.5 & 748.7 \\
$750: 500$ Polyrhythm & & & \\
Finger (750ms) & 745.8 & 843.6 & 747.7 \\
Foot (500ms) & 512.0 & 616.0 & 499.2 \\
$500: 750$ Polyrhythm & & & \\
Finger (500ms) & 509.4 & 608.6 & 498.9 \\
Foot (750ms) & 775.1 & 783.2 & 748.1 \\
\hline
\end{tabular}

\section{Discussion}

This research compared the ability to coordinate limbs with external rhythms among participants with different backgrounds of sports and musical experiences, i.e., having experiences in all limbs moving with external rhythm (Ds), having experiences in only a part of limb coordinating with external rhythm (ASs), and no experiences in moving limbs with external rhythm (WPs). The results indicated no statistically significant differences on any variable (PCRC, SDS, or $\mathrm{CV}_{\text {ITI }}$ ) among the three groups on the single-rhythm task. While Ds might have had an advantage in our experimental task due to their nature of the practice, the results of single rhythmic task showed that this task was simple enough for all participants regardless of their background. 
On polyrhythmic tasks, the WPs were outperformed by the ASs and Ds on every variable, whereas there was no significant difference between ASs and Ds in PCRC and $\mathrm{CV}_{\text {ITI. }}$ These findings implied that polyrhythmic conditions were more difficult than a single-rhythm task, as suggested by the extant literature (Kurtz \& Lee, 2003; Peper \& Beek, 1998; Starke \& Baber, 2017; Summers \& Kennedy, 1992; Summers, Rosenbaum, et al., 1993; Summers, Todd, et al., 1993). Although neither the ASs nor the WPs in our study had much musical experience, artistic swimming training always involves music because of the nature of artistic swimming competitions. However, WPs had neither substantial musical experience nor involvement with music during their athletic training. It is probable that such differences in daily training routines caused the difference in the ability between the groups. Our results agree with the finding in extant literature that musical experiences improve limb coordination on complex polyrhythmic tasks with auditory cueing tasks (Klapp, 1979; Summers \& Kennedy, 1992; Summers, Rosenbaum, et al., 1993; Summers, Todd, et al., 1993).

However, there was no difference between Ds and ASs on these variables, even though Ds are probably more experienced in complex movements than ASs because Ds move all their limbs with musical rhythms whereas ASs coordinate only some of their limbs with musical rhythms at any given time. Since the above-water movements (either upper or lower limb movements) were dictated by music but not the underwater movements, ASs were probably familiar with moving upper and lower limbs separately from one another. This common practice of ASs was similar to Ds where they needed to coordinate their two hands and two feet with different rhythms. Even though WPs also seems to move their limbs independently similar to ASs, the background mechanism might differ between ASs and WPs. In water polo, the motor skills were related to ball manipulations and controls. Their movements of upper and lower limbs must be 
coordinated to produce an effective kinetic chain to generate powerful shots or to control passes (Weber, Kontaxis, O'Brien, \& Bedi, 2014). Hence, it might be that ASs and Ds have good ability to produce polyrhythmic limb coordination due to requirements in training and performance where limb coordination with music is essential.

It should also be noted that the standard deviation of PCRC and ITI of ASs were grater than Ds. Every participant in D group performed much consistently with one another, while some participants in AS group could not perform consistently with their peers. This result was consistent with SDS where Ds felt more comfortable with the polyrhythmic tasks than ASs and WPs. Given that people with a good musical ability and experience had low tapping variability than those with limited experience (Iannarilli, Vannozzi, Iosa, Pesce, \& Capranica, 2013). This was probably due to greater musical experiences of Ds compared with ASs. Considering the nature of Ds' daily training and performance, Ds might have been more familiar with the experimental tasks than ASs.

Although the 750:500 and 500:750 tasks were set up as polyrhythmic, both entailed a syncopated rhythm in which the desired ITI between finger and foot was exactly $250 \mathrm{~ms}$. Hence, it would be easier to complete the sequence of the polyrhythmic tasks if the participants treated it as an isochronous sequence between finger and foot. Prior studies (Fidali, Poudrier, \& H Repp, 2011; Pressing, Summers, \& Magill, 1996; Summers, Ford, et al., 1993; Summers \& Kennedy, 1992; Summers, Rosenbaum, et al., 1993; Summers, Todd, et al., 1993) suggested that syncopated rhythms were easier to execute as a sequentially integrated rhythmic pattern, i.e. using an integrated timing strategy. To successfully implement an integrated timing strategy, conscious counting is required (Krampe, Kliegl, Mayr, Engbert, \& Vorberg, 2000). Integrated timing was a common practice for musicians especially in drummers (Jagacinski et al., 2016), while 
silent counting was required by ASs (Bespalov \& Leonov, 2012; Leonov, 2012). The similarity between ASs and Ds can be explained by the tendency for both groups to develop such strategies through their daily training, and the results of the polyrhythmic tasks highlight the importance of applying a conscious counting technique and rhythmic understanding when completing a complex rhythmic task (Bespalov \& Leonov, 2012).

While our results suggested a possibility that music experiences affect the coordination of the limbs with external rhythms, some limitations should be noted. Firstly, our study did not have a large sample number in each group. However, due to the high expertise of participants (e.g., international level athletes), the expected whole population of each group (i.e., elite ASs, WPs, Ds) would also be very small. Therefore, even though the absolute number is small, eight samples per elite group are very valuable and this does not necessarily mean that the rate of sample size relative to the whole population is also small. The second potential limitation is the gender effect. Due to the requirements and characteristics of artistic swimming, water polo, and drumming, the groups tested in the present study were not well balanced in gender, which might have influenced the results as suggested by Poudrier (2018). However, Aoki, Furuya, and Kinoshita (2005) and $\mathrm{Au}, \mathrm{Seah}, \mathrm{Li}$, and Tan (2015) reported that gender had no influence on tapping ability. This inconsistency in the literature means that the effect of gender is still unknown and need further investigation. It should also be emphasised that the three groups have different natures of motor skills used in daily training and performances. Lastly, our experiment was a unilateral movement of upper and lower extremities in contrast to bimanual movements discussed in the literature. While neurophysiologists suggested that bimanual movements required utilisation of both sides of the corpus callosum, several studies suggested that a fine and delicate tapping also requires utilisation of corpus callosum (Beaulé et al., 2012; Vuust et al., 2011). As 
neurophysiological mechanism was not a focus of this study, further investigation in terms of the neurophysiology of unilateral tapping of upper and lower extremity differences between the groups would of interest.

\section{Conclusion}

In this study, we investigated polyrhythmic production ability among participants with experience in complex inter-limb coordination tasks but with different musical backgrounds in music: experience in coordinating all limb movements with music (Ds), only some limb movements with music (ASs), or no limb movements in synchronisation with music (WPs). We found that the three groups of participants exhibited no statistically significant differences of the investigated variables in the single-rhythm task (PCRC, SDS, and $\mathrm{CV}_{\text {ITI }}$ ), but the PCRC and $\mathrm{CV}_{\text {ITI }}$ of WPs were significantly different from those of ASs and Ds in the polyrhythmic tasks. No significant differences of PCRC and CV ITI were found between the ASs and Ds. The results emphasise the importance of limb coordination with music in enabling polyrhythmic production, regardless of the number of limbs that must be coordinated with music in one's daily training.

\section{Acknowledgement}

This research was supported by JSPS KAKENHI Grant Number JP17K01716. We are grateful to all members of the Swimming Laboratory at the University of Tsukuba for their helpful advice. In addition, we thank all the athletes and musicians who participated in this study. 


\section{References}

Aoki, T., Furuya, S., \& Kinoshita, H. (2005). Finger-tapping ability in male and female pianists and nonmusician controls. Motor Control, 9(1), 23-39. doi:10.1123/mcj.9.1.23

Au, W.-L., Seah, I., Li, W., \& Tan, L. (2015). Effects of Age and Gender on Hand Motion Tasks. Parkinson's Disease, 2015, 1-5. doi:10.1155/2015/862427

Beaulé, V., Tremblay, S., \& Théoret, H. (2012). Interhemispheric Control of Unilateral Movement. Neural plasticity, 2012, 627816. doi:10.1155/2012/627816

Bespalov, B. I., \& Leonov, S. V. (2012). Characteristics of silent counting in synchronized swimmers. Psychology in Russia, 5, 498.

Bianco, V., Berchicci, M., Perri, R. L., Quinzi, F., \& Di Russo, F. (2017). Exercise-related cognitive effects on sensory-motor control in athletes and drummers compared to non-athletes and other musicians. Neuroscience, 360, 39-47. doi:https://doi.org/10.1016/j.neuroscience.2017.07.059

Buhmann, J., Moens, B., Van Dyck, E., Dotov, D., \& Leman, M. (2018). Optimizing beat synchronized running to music. PLoS One, 13(12), e0208702. doi:10.1371/journal.pone.0208702

Clayton, M., Sager, R., \& Will, U. (2004). In Time With the Music: The Concept of Entrainment and Its Significance for Ethnomusicology. European Meetings in Ethnomusicology, 11 . 
Cohen, J., Cohen, P., West, S. G., \& Aiken, L. S. (Producer). (2003). Applied multiple regression/correlation analysis for the behavioral sciences, 3rd ed. Applied multiple regression/correlation analysis for the behavioral sciences, $3 r d$ ed.

Deutsch, D. (1978). Chapter 9 - THE PSYCHOLOGY OF MUSIC**This work was supported by United States Public Health Service Grant MH-21001-03. In E. C. Carterette \& M. P. Friedman (Eds.), Perceptual Ecology (pp. 191-224): Academic Press.

Deutsch, D. (1996). Chapter 8 The perception of auditory patterns. In W. Prinz \& B. Bridgeman (Eds.), Handbook of Perception and Action (Vol. 1, pp. 253-296): Academic Press.

Fidali, B., Poudrier, E., \& H Repp, B. (2011). Detecting perturbations in polyrhythms: Effects of complexity and attentional strategies. Psychological research, 77. doi:10.1007/s00426-011-0406-8

Iannarilli, F., Vannozzi, G., Iosa, M., Pesce, C., \& Capranica, L. (2013). Effects of task complexity on rhythmic reproduction performance in adults. Hum Mov Sci, 32(1), 203-213. doi:10.1016/j.humov.2012.12.004

Jagacinski, R. J., Kim, T. H., \& Lavender, S. A. (2009). Managing the rhythmic complexity of hitting a golf ball. Journal of Motor Behavior, 41(5), 469-477. doi:10.3200/35-08-075

Jagacinski, R. J., Rizzi, E., Kim, T. H., Lavender, S. A., Speller, L. F., \& Klapp, S. T. (2016). Parallel streams versus integrated timing in multilimb pattern generation: 
A test of Korte's Third Law. J Exp Psychol Hum Percept Perform, 42(11), 17031715. doi:10.1037/xhp0000263

Jin, X., Biye, W., Lv, Y., Lu, Y., Chen, J., \& Zhou, C. (2019). Does dance training influence beat sensorimotor synchronization? Differences in finger-tapping sensorimotor synchronization between competitive ballroom dancers and nondancers. Experimental Brain Research, 237. doi:10.1007/s00221-018-5410-4

Karpati, F. J., Giacosa, C., Foster, N. E., Penhune, V. B., \& Hyde, K. L. (2016). Sensorimotor integration is enhanced in dancers and musicians. Exp Brain Res, 234(3), 893-903. doi:10.1007/s00221-015-4524-1

Klapp, S. T. (1979). Doing two things at once: The role of temporal compatibility. Memory \& Cognition, 7(5), 375-381. doi:10.3758/BF03196942

Krampe, R. T., Kliegl, R., Mayr, U., Engbert, R., \& Vorberg, D. (2000). The fast and the slow of skilled bimanual rhythm production: parallel versus integrated timing. $J$ Exp Psychol Hum Percept Perform, 26(1), 206-233.

Krause, V., Pollok, B., \& Schnitzler, A. (2010). Perception in action: the impact of sensory information on sensorimotor synchronization in musicians and nonmusicians. Acta Psychol (Amst), 133(1), 28-37. doi:10.1016/j.actpsy.2009.08.003

Kurtz, S., \& Lee, T. D. (2003). Part and whole perceptual-motor practice of a polyrhythm. Neuroscience Letters, 338(3), 205-208. doi:10.1016/s0304-3940(02)01394-0

Leonov, S. (2012). Diagnostics of time perception in synchronized swimming. Procedia Social and Behavioral Sciences, 33, 771-775. doi:https://doi.org/10.1016/j.sbspro.2012.01.226 
Maes, P.-J., Lorenzoni, V., \& Six, J. (2018). The SoundBike: musical sonification strategies to enhance cyclists' spontaneous synchronization to external music. Journal on Multimodal User Interfaces. doi:10.1007/s12193-018-0279-x

Martins, M., Neves, L., Rodrigues, P., Vasconcelos, O., \& Castro, S. L. (2018). OrffBased Music Training Enhances Children's Manual Dexterity and Bimanual Coordination. Front Psychol, 9, 2616. doi:10.3389/fpsyg.2018.02616

Mo, S., \& Chow, D. (2018). Differences in lower-limb coordination and coordination variability between novice and experienced runners during a prolonged treadmill run at anaerobic threshold speed. Journal of Sports Sciences, 37, 1-8. doi:10.1080/02640414.2018.1539294

Park, S.-W., \& Sternad, D. (2015). Robust retention of individual sensorimotor skill after self-guided practice. Journal of neurophysiology, 113(7), 2635-2645. doi:10.1152/jn.00884.2014

Peper, C., \& Beek, P. (1998). Distinguishing between the effects of frequency and amplitude on interlimb coupling in tapping a 2:3 polyrhythm. Experimental brain research. Experimentelle Hirnforschung. Expérimentation cérébrale, 118, 78-92. doi:10.1007/s002210050257

Poudrier, È. (2018). Tapping to Carter: Mensural Determinacy in Complex Rhythmic Sequences. Empirical Musicology Review, 12, 277. doi:10.18061/emr.v12i34.5814 
Pressing, J., Summers, J., \& Magill, J. (1996). Cognitive multiplicity in polyrhythmic pattern performance. [doi:10.1037/0096-1523.22.5.1127]. US: American Psychological Association.

Repp, B. H. (2010). Sensorimotor synchronization and perception of timing: effects of music training and task experience. Hum Mov Sci, 29(2), 200-213. doi:10.1016/j.humov.2009.08.002

Repp, B. H., \& Su, Y.-H. (2013). Sensorimotor synchronization: A review of recent research (2006-2012). Psychonomic Bulletin \& Review, 20(3), 403-452. doi:10.3758/s13423-012-0371-2

Seifert, L., Leblanc, H., Chollet, D., \& Delignières, D. (2010). Inter-limb coordination in swimming: Effect of speed and skill level. Human Movement Science, 29(1), 103113. doi:https://doi.org/10.1016/j.humov.2009.05.003

Seifert, L., Leblanc, H., Herault, R., Komar, J., Button, C., \& Chollet, D. (2011). Interindividual variability in the upper-lower limb breaststroke coordination. Hum Mov Sci, 30(3), 550-565. doi:10.1016/j.humov.2010.12.003

Shaffer, L. H. (1981). Performances of Chopin, Bach, and Bartok - Studies in Motor Programming. Cognitive Psychology, 13(3), 326-376. doi:Doi 10.1016/00100285(81)90013-X

Sommer, M., Hager, C. K., Boraxbekk, C. J., \& Ronnqvist, L. (2018). Timing Training in Female Soccer Players: Effects on Skilled Movement Performance and Brain Responses. Front Hum Neurosci, 12, 311. doi:10.3389/fnhum.2018.00311 
Starke, S. D., \& Baber, C. (2017). Spontaneous bimanual independence during parallel tapping and sawing. Plos One, 12(5). doi:ARTN e0178188

10.1371/journal.pone.0178188

Summers, J. J., Ford, S. K., \& Todd, J. A. (1993). Practice effects on the coordination of the two hands in a bimanual tapping task. [doi:10.1016/0167-9457(93)90039-R]. Netherlands: Elsevier Science.

Summers, J. J., \& Kennedy, T. M. (1992). Strategies in the production of a 5:3 polyrhythm. Human Movement Science, 11(1-2), 101-112. doi:10.1016/0167-9457(92)90053$\mathrm{E}$

Summers, J. J., Rosenbaum, D. A., Burns, B. D., \& Ford, S. K. (1993). Production of Polyrhythms. Journal of Experimental Psychology-Human Perception and Performance, 19(2), 416-428. doi:Doi 10.1037/0096-1523.19.2.416

Summers, J. J., Todd, J. A., \& Kim, Y. H. (1993). The Influence of Perceptual and Motor Factors on Bimanual Coordination in a Polyrhythmic Tapping Task. Psychological Research-Psychologische Forschung, 55(2), 107-115. doi:Doi 10.1007/Bf00419641

Tajima, M., \& Choshi, K. (2000). Effects of learning and movement frequency on polyrhythmic tapping performance. Perceptual and motor skills, 90, 675-690. doi:10.2466/PMS.90.2.675-690

van de Rijt, A. (2018). All-sense-all networks are suboptimal for sensorimotor synchronization. PLoS One, 13(8), e0202056. doi:10.1371/journal.pone.0202056 
Vuust, P., Wallentin, M., Mouridsen, K., Ostergaard, L., \& Roepstorff, A. (2011). Tapping polyrhythms in music activates language areas. Neurosci Lett, 494(3), 211-216. doi:10.1016/j.neulet.2011.03.015

Weber, A. E., Kontaxis, A., O'Brien, S. J., \& Bedi, A. (2014). The biomechanics of throwing: simplified and cogent. Sports Med Arthrosc Rev, 22(2), 72-79. doi:10.1097/jsa.0000000000000019

Yokus, H., \& Yokus, T. (2015). Polyrhythmic Tapping: Examining the Effectiveness of the Strategy of Organizing Rhythmic Structures through Synthesis. Educational Sciences: Theory \& Practice, 15, 239-252. doi:10.12738/estp.2015.1.1917 